\title{
N92-21948
}

\section{Prompt Particle Acceleration around Moving X-point Magnetic Field during Impulsive Phase of Solar Flares}

\author{
Jun-ichi Sakai \\ Laboratory for Plasma Astrophysics and Fusion Science \\ Department of Electronics and Information \\ Faculty of Engineering, Toyama University \\ Toyama 930 JAPAN
}

\begin{abstract}
We present a model for high-energy solar flares to explain prompt proton and electron acceleration, which occurs around moving $\mathrm{X}$-point magnetic field during the implosion phase of the current sheet. We derive the electromagnetic fields during the strong implosion of the current sheet, which is driven by the converging flow toward the center of the magnetic arcade. We investigated test particle motion in the strong electromagnetic fields derived from the MHD equations. It is shown that both protons and electrons can be promptly(within $1 \mathrm{~s}$ ) accelerated to $\sim 70 \mathrm{MeV}$ and $\sim 200 \mathrm{MeV}$, respectively. This acceleration mechanism can be applicable for the impulsive phase of the gradual gamma ray and proton flares(gradual GR/P flare), which have been called $t w o$ ribbon flares.
\end{abstract}

\section{Introduction}

One of the important problems in high-energy solar flare physics is to understand the physical mechanism(s) of high energy particle acceleration during solar flares. From the observations by SMM and Hinotori satellites, it became clear that there are two classes of gamma-ray/proton(GR/P) flares- impulsive GR/P flares and gradual GR/P flares( see, for a review , Bai and Sturrock 1989). The GR/P flares refer to flares that produce nuclear gamma-rays and /or energetic interplanetary protons. Most short flares $(<100 \mathrm{~s})$ corresponds to be impulsive GR/P flares, while most of the long-duration flares $(>200 \mathrm{~s}$ ) corresponds to the gradual GR/P flares. In the impulsive GR/P flares there are two phases of particle acceleration : the first phase is that both electrons and protons are accelerated to $\sim 10 \mathrm{MeV}$ and to $\sim 100 \mathrm{MeV}$ energies, respectively, within $1 \mathrm{~s}$ (Kane et al. 1986; Riger 1989), and second phase is that electrons up to $100 \mathrm{MeV}$ and protons up to $\mathrm{GeV}$ energies are accelerated within a few seconds to $100 \mathrm{~s}$.

In order to explain the above prompt particle acceleration to relativistic energies, Sakai(1990) showed that during the 3D X-type current loop coalescence, where two crossed flux tubes interact in one point, both protons and electrons can be promptly(within less than $1 \mathrm{~s}$ ) accelerated to $\sim 100 \mathrm{GeV}$ and $\sim 100 \mathrm{MeV}$, respectively. De Jager and Sakai (1991) showed that the burst duration of elementary flare bursts(5-25 s) observed during the impulsive GR/P flares can be explained quantitatively by the mechanism of the 3D X-type current loop coalescence.

In the present paper we show a model for the long-duration gamma ray /proton flare(the gradual GR/P flares) to explain prompt proton and electron acceleration during the impulsive phase. These flares have been called $t w o-$ ribbon flares and almost all gradual GR/P flares show impulsive behaviour in the beginning of the hard X-ray emission and gradual behaviour later on (Bai and Sturrock 1989 ). In most large two-ribbon flares with filament eruptions the filament begins to move up several minutes or more before the onset of the impulsive phase(Martin and Ramsey 1972; Kahler et al. 1988). Recently Sakai and Koide (1991) presented a theory of the filament eruption before the impulsive phase of solar flares. Figure 1 shows a schematic picture of the global magnetic field configuration and the filament which is located above the $\mathrm{X}$-point magnetic configuration near the center of the magnetic arcade. Sakai and Koide (1991) showed that the upward motion of the X-point which may trace the filament eruption begins several minutes before the impulsive phase where the magnetic field, $\mathrm{Bx}$ produced by the current near the $\mathrm{X}$-point can exceed the magnetic field, $\mathrm{Bz}$ along the filament. If the $\mathrm{Bx}$ exceeds the $\mathrm{Bz}$, then the explosive magnetic reconnection (Tajima 1982; Sakai et al. 1984; Sakai and Ohsawa 1987) can be triggered in the current sheet. During the implosion phase of the current sheet, the strong electric field can be induced by the strong converging flow around the $\mathrm{X}$-point.

In Section 2 we drive the electromagnetic fields during the implosion phase of the current sheet from the MHD equation. In Section 3 we investigate a test proton and electron motion under the electromagnetic fields derived in the previous section. We will show that both protons and electrons can be promptly accelerated within $1 \mathrm{~s}$ to $\sim 70$ $\mathrm{MeV}$ and $\sim 200 \mathrm{MeV}$, respectively. In Section 4 we summarize our results. 


\section{Electromagnetic Fields around Moving X-point}

\subsection{MHD Equation and Energy Equation}

In this section we present basic equations describing the dynamics of the current sheet near the magnetic X-point, which is located under the filament as shown in Figure 1. We assume that the filament is supported in the upper part of the X-type magnetic field along the $\mathrm{z}$-direction, in which there exists the magnetic field, Bz. Following the gradual shearing motion at the footpoint of the magnetic arcade, the plasma upward motion can be induced, and the current near the $\mathrm{X}$-point can be increased due to the converging flow near the current sheet. The current sheet continues to elongate to the vertical direction, and to become thin in the horizontal direction. We will focus on the dynamics of the plasma around the $X$-point to determine the electromagnetic fields during thinning of the current sheet. The $\mathrm{x}$-axis is taken in the vertical direction, and the $\mathrm{y}$-axis is in the horizontal direction. The current sheet is assumed to be homogeneous in the horizontal z-direction.

We begin from the following MHD equations including the gravity and Joule heating effect in the energy equation;

$$
\begin{aligned}
& \frac{\partial \rho}{\partial t}+\nabla \cdot(\rho \mathbf{v})=0 \\
& \rho\left(\frac{\partial \mathbf{v}}{\partial t}+\mathbf{v} \cdot \nabla \mathbf{v}\right)=-\nabla P+\frac{1}{4 \pi}(\nabla \times \mathbf{B}) \times \mathbf{B}-\rho g \vec{e}_{x} \\
& \frac{\partial \mathbf{B}}{\partial t}=\nabla \times(\mathbf{v} \times \mathbf{B})+\frac{c^{2}}{4 \pi \sigma} \Delta \mathbf{B}, \\
& \frac{\partial P}{\partial t}+(\mathbf{v} \cdot \nabla) P+\gamma P \nabla \cdot \mathbf{v}=(\gamma-1) \frac{(\nabla \times \mathbf{B})^{2}}{16 \pi^{2} \sigma},
\end{aligned}
$$

where $r, v, P, B$ are the density, velocity, pressure, and magnetic field, respectively, and $\gamma$ is the adiabatic constant. The gravitational acceleration is given by

$$
g(x)=g_{0}\left(1+\frac{x^{2}}{R^{2}}\right)^{-2}
$$

where $\mathrm{R}=\mathrm{R}_{\mathrm{S}}+\mathrm{r}_{0}$ and $\mathrm{g}_{0}=G_{\mathrm{S}} \mathrm{R}^{-2} \cdot \overrightarrow{\mathrm{M}}_{\mathrm{S}}, \mathrm{R}_{\mathrm{S}}$ and $\mathrm{r}_{0}$ are the solar mass, solar radius and distance to the origin of the coordinate from the solar surface, respectively. The right-hand side in Equation (4) shows the effect of the Joule heating.

\subsection{Electromagnetic Fields around the Current Sheet}

During the gradual shearing motion of the photosphere, the current near the magnetic $\mathrm{X}$-point can increase due to horizontal plasma inflows from both sides. This horizontal plasma flow, $v_{y}$ around the $\mathrm{X}$-point can be approximately given by

$$
v_{y}=\left(\frac{1}{a}\right)\left(\frac{d a}{d t}\right) y
$$

where $a(t)$ is a time dependent scale factor. The scale factor $a(t)$, which is determined later, characterizes continuous change of thickness of the current sheet due to the plasma inflows. The vertical flow, $v_{X}$ is taken to be 


$$
v_{x}=v_{x 0}+\left(\frac{1}{b} \frac{d b}{d t}\right) x \text {, }
$$

where $\mathrm{vxO}_{\mathrm{O}}(\mathrm{t})$ and another scale factor $\mathrm{b}(\mathrm{t})$ are determined self-consistently later.

The components of the magnetic field are taken as

$$
\begin{aligned}
& B_{x}=B_{x 0}(t) \frac{y}{\lambda}, \\
& B_{y}=B_{n}(t)+B_{y 0}(t) \frac{x}{\lambda}, \\
& B_{z}=B_{z 0}(t),
\end{aligned}
$$

where $\lambda$ is a characteristic scale-length of the current sheet, which can be considered to be the same as the radius of the filament located above the $X$-point. The magnetic field $B_{n}$ in Equation (9) shows a role that the $X$-point can move during the evolution of the current sheet. Unknown functions, $\mathrm{B}_{\mathbf{x} 0}, \mathrm{~B}_{\mathbf{n}}, \mathrm{B}_{\mathbf{y} 0}$ and $\mathrm{B}_{\mathrm{z} 0}$ can be determined, selfconsistently later.

Substituting Equations (6) and (7) into Equation (1), we find that the density $\rho(t)$ is only a function of time and given by

$$
\rho(t)=\frac{\rho_{0}}{a(t) b(t)}
$$

where $\rho_{0}$ is a constant.

From the induction equation (3), using the expression for the magnetic fields (8)-(10) and velocities (6) and (7), we find

$$
\begin{aligned}
& B_{x 0}=\frac{B_{0}}{a^{2}} \\
& B_{y 0}=\frac{B_{0}}{b^{2}}, \\
& B_{z 0}=\frac{B_{00}}{a b}, \\
& \frac{d B_{n}}{d t}+\frac{v_{x 0}}{\lambda} \frac{B_{0}}{b^{2}}+B_{n} \frac{1}{b}\left(\frac{d b}{d t}\right)=0
\end{aligned}
$$

where $\mathrm{B}_{0}$ and $\mathrm{B}_{00}$ are constants.

We assume the pressure $P(x, y, t)$ to be

$$
P(x, y, t)=P_{00}(t)-P_{0}(t) \frac{x}{\lambda}-P_{x 0}(t)\left(\frac{x}{\lambda}\right)^{2}-P_{y 0}(t)\left(\frac{y}{\lambda}\right)^{2} .
$$

We here summarize normalized basic equations, which will be numerically solved in the next section, 


$$
\begin{aligned}
\frac{d^{2} a}{d t^{2}} & =\frac{\beta_{p}}{a^{\gamma} b^{\gamma-1}}+\left(\frac{1}{b}-\frac{b}{a^{2}}\right) \\
\frac{d^{2} b}{d t^{2}} & =\frac{\beta_{p}}{a^{\gamma-1} b^{\gamma}}-\left(\frac{a}{b^{2}}-\frac{1}{a}\right)+G_{1} b \\
\frac{d B_{n 0}}{d t} & =-\frac{v_{x 0}}{b^{2}}-B_{n 0} \frac{1}{b} \frac{d b}{d t}, \\
\frac{d v_{x 0}}{d t} & =-v_{x 0} \frac{1}{b} \frac{d b}{d t}+\beta_{p} a b P_{0}-a b B_{n 0}\left(\frac{1}{b^{2}}-\frac{1}{a^{2}}\right)-G_{2}, \\
\frac{d P_{0}}{d t} & =-P_{0} \frac{1}{b} \frac{d b}{d t}-\frac{2 v_{x 0}}{a^{\gamma} b^{\gamma+2}}-\gamma P_{0}\left(\frac{1}{a} \frac{d a}{d t}+\frac{1}{b} \frac{d b}{d t}\right), \\
\frac{d P_{00}}{d t} & =v_{x 0} P_{0}-\gamma P_{00}\left(\frac{1}{a} \frac{d a}{d t}+\frac{1}{b} \frac{d b}{d t}\right)+(\gamma-1) \frac{\beta_{p}}{S}\left(\frac{1}{b^{2}}-\frac{1}{a^{2}}\right)^{2},
\end{aligned}
$$

where $\beta_{p}=c_{s}{ }^{2} / v_{\text {ap }} 2$ is plasma beta-ratio, which is determined from the magnetic field, $B_{0}$ produced by the current induced near the $X$-point. The magnetic Reynolds number, $S$ is $S=\tau_{B} / \tau_{A}\left(\tau_{B}=4 \pi \sigma \lambda^{2} / c^{2}\right)$. The parameters $G_{1}$ in Equation (18) and $G_{2}$ in Equation (20) are given as

$$
\begin{gathered}
G_{1}=\frac{2 g_{0} \tau_{A}^{2}}{R}, \\
G_{2}=\frac{g_{0} \tau_{A}}{v_{a p}} .
\end{gathered}
$$

In the above equations (17)-(22), the time $, \mathrm{B}_{\mathrm{n} 0}, \mathrm{v}_{\mathrm{x} 0}, \mathrm{P}_{0}, \mathrm{P}_{00}$ are normalized by $\tau_{\mathrm{A}}=\lambda /$ vap, $\mathrm{B} 0$, vap, $\mathrm{Pa}$ and $\mathrm{Pa}$, respectively.

The normalized time-dependent part of the magnetic fields, $\mathrm{Bx}$ and $\mathrm{Bz}$ are given by

$$
\begin{aligned}
& B_{x a}=\frac{1}{a^{2}}, \\
& B_{z a}=R_{b} \frac{1}{a b},
\end{aligned}
$$

where $\mathrm{R}_{b}=\mathrm{B} \mathrm{Bo}_{\mathrm{B}} \mathrm{B}$ o gives the initial ratio between $\mathrm{Bz}$ and $\mathrm{By}$.

The electric field $\mathbf{E}$ induced by the change of the magnetic field $B($ eqs. [8]-[10] ) can be determined from

$$
\mathbf{E}=-\mathbf{v} \times \mathbf{B} / \mathbf{c} \text {. }
$$

By use of equations (6)-(10), we obtain the components of the inductive electric field; 


$$
\begin{aligned}
& E_{x}=-\frac{B_{z 0}}{c a^{2} b} \frac{d a}{d t} y \\
& E_{y}=\frac{B_{z 0}}{c a b^{2}} \frac{d b}{d t} x+\frac{v_{x 0}}{c} \frac{B_{z 0}}{a b} \\
& E_{z}=\frac{B_{0}}{c}\left[\frac{1}{a^{3}} \frac{d a}{d t} \frac{y^{2}}{\lambda}-\frac{1}{b^{3}} \frac{d b}{d t} \frac{x^{2}}{\lambda}-\frac{v_{x 0} B_{n 0}}{B_{0}}-\frac{v_{x 0}}{b^{2}} \frac{x}{\lambda}\right. \\
& \left.-\frac{B_{n 0}}{B_{0}} \frac{1}{b} \frac{d b}{d t}\right]
\end{aligned}
$$

We can obtain numerical results of the electromagnetic fields by solving the basic equations (17)-(20). The initial conditions are taken as $\mathrm{a}=1, \mathrm{da} / \mathrm{dt}=-0.001, \mathrm{~b}=2, \mathrm{db} / \mathrm{dt}=0, \mathrm{~B}_{\mathbf{n}}=0.01, \mathrm{v}_{\mathbf{x} 0}=0.001$, and other parameters are taken as $G_{1}=0.01, G_{2}=0.27, S=10^{5}$, and $R_{b}=B_{00} / B_{0}=100 / 10$. The plasma beta-ratio $\beta_{p}$ is 1.0 and the adiabatic ratio is $\gamma=5 / 3$.

Some components of the inductive electric fields become explosively strong near the time $1.18 \tau_{\mathrm{A}}$. The reason is due to the fact that near the implosion phase, the converging flow, $v y$ is increased by about 100 times and the magnetic field, $B_{x a}$ is also increased by about 150 times through the current pinch. Therefore, the inductive electric field $\mathrm{E}_{\mathbf{X}}=\mathrm{v}_{\mathbf{y}} \mathrm{B}_{\mathbf{x}} 0^{/ \mathrm{c}}$ can be amplified to $1.5 \times 10^{4}$ times, compared with the initial values. This inductive electric field before the implosion is about $70 \mathrm{~V} / \mathrm{m}$, if we take $v_{a p}=7 \times 10^{6} \mathrm{~cm} / \mathrm{s}$ and $B_{0}=10 \mathrm{G}$. The inductive electric field near the implosion can become $E_{\mathbf{X}} \sim 10^{6} \mathrm{~V} / \mathrm{m}$. This strong electric field near the implosion is important for the high-energy particle acceleration. If we take $\lambda=2 \times 10^{9} \mathrm{~cm}$, the Alfven transit time $\tau_{\mathrm{A}}$ is $\tau_{\mathrm{A}}=\lambda \mathrm{v}_{\text {ap }}=300 \mathrm{~s}$. Then the duration of implosion is about $0.01 \tau_{A}=3 \mathrm{~s}$. As seen in the next section, the acceleration time for both protons and electrons is quite rapid compared with the duration of implosion , $3 \mathrm{~s}$. Therefore we will investigate the motion of a test particle under the electromagnetic fields (28)-(30) in the next section.

\subsection{Equation of Motion}

\section{Proton and Electron Acceleration}

We consider the motion of a test proton under the electromagnetic fields given by equations (28)-(30)

$$
\begin{aligned}
& \frac{d \tilde{\mathbf{P}}}{d t}=\left(\frac{v_{a p}}{c} \tilde{\mathbf{E}}+\frac{\tilde{\mathbf{P}} \times \tilde{\mathbf{B}}}{\Gamma_{p}}\right), \\
& \frac{d \tilde{x}}{d \tilde{t}}=R_{p} \frac{\tilde{P}_{x}}{\Gamma_{p}}, \\
& \frac{d \tilde{y}}{d \tilde{t}}=R_{p} \frac{\tilde{P}_{y}}{\Gamma_{p}}, \\
& \frac{d \tilde{z}}{d \tilde{t}}=R_{p} \frac{\tilde{P}_{z}}{\Gamma_{p}},
\end{aligned}
$$

where $\tilde{\mathbf{P}}=\mathbf{P} / \mathrm{m}_{\mathrm{p}} \mathrm{c}, \tilde{\mathbf{E}}=\mathbf{E} / \mathrm{E}_{0}, \tilde{\mathbf{B}}=\mathbf{B} / \mathrm{B}_{0}, \mathrm{R}_{\mathrm{p}}=\mathrm{c} / \mathrm{atp}_{\mathrm{c}} \lambda, \tilde{\mathrm{x}}=\mathrm{x} / \lambda, \tilde{\mathrm{y}}=\mathrm{y} / \lambda, \tilde{z}=\mathrm{z} / \lambda$ The time is normalized by the proton cyclotron period $\omega^{-1} \mathrm{cp}\left(\tilde{\mathrm{t}}=\omega_{\mathrm{cp}} \mathrm{t}, \omega_{\mathrm{cp}}=\mathrm{eB} \mathrm{B}_{0} / \mathrm{m}_{\mathrm{p}} \mathrm{c}\right)$. 


\subsection{Numerical Results for Proton Acceleration}

The time-dependent amplitudes of the electromagnetic fields are assumed to be constant during the acceleration time, because the typical acceleration time to relativistic energy for the proton is about $10^{5} \omega^{-1} \mathrm{cp}$, which is about $1 \mathrm{~s}$ for $\mathrm{B}_{0}=10 \mathrm{G}$ and is still within the duration of implosion, $\sim 0.01 \tau_{\mathrm{A}}=3 \mathrm{~s}$. We investigate the motion of the proton at the time, $t=1.168 \tau_{\mathrm{A}}$. The electromagnetic field amplitudes at the time, $t=1.168 \tau_{\mathrm{A}}$ are $\mathrm{B}_{\mathrm{xa}}=119.6$, $\mathrm{B}_{\mathrm{ya}}=0.11, \mathrm{~B}_{\mathbf{Z a}}=37.0, \mathrm{~B}_{\mathrm{n}}=-0.11, \mathrm{EX}_{1}=-2908.28, \mathrm{EY}_{0}=35.5, \mathrm{EY}_{1}=25.5, \mathrm{EZ}_{1}=0.079, \mathrm{EZ}_{2}=-9391.1, \mathrm{EZ}_{0}$ $=-0.113$ and $\mathrm{EZ}=0.029$. We take $\mathrm{R}_{\mathrm{p}}=\mathrm{c} / \omega_{\mathrm{cp}} \lambda=10^{-5}$ and $\mathrm{v}_{\mathrm{ap}} / \mathrm{c}=1 / 4300$. We show the results for the initial conditions with $x(0)=y(0)=z(0)=0.5, P_{x}=P_{y}=P_{z}=10^{-4}$. Figure 2 shows the time history of the Lorentz factor $\Gamma=\left(1+\mathrm{P}^{2}\right)^{1 / 2}$ for the proton. Figure 3 shows the time history of the momentum for the proton. The proton can be accelerated to the $x$-and $y$-direction. As seen in Figure 3 , the average value of the momentum $P_{x}$ increases almost proportional to the time. Therefore we can evaluate the time dependency of the momentum $P_{x}$ as

$$
\tilde{P}_{x}=2.5 \times 10^{-4} \omega_{c p} t+10^{-4}
$$

for $y(0)=0.5$. Similarly, we find

$$
\tilde{P}_{x}=1.0 \times 10^{-5} \omega_{c p} t+10^{-4}
$$

for $y(0)=0.1$ and

$$
\tilde{P}_{x}=4.0 \times 10^{-6} \omega_{c p} t+10^{-4}
$$

for $y(0)=0.05$. These results are almost same for the initial momentum in the range of $P(0)=10^{-6}$ to $10^{-4}$ as well as for the other initial positions, $x(0)$ and $z(0)$. Therefore we conclude that within about one second of the implosion time the proton can be accelerated to $\sim 70 \mathrm{MeV}$ for protons with $\mathrm{y}(0)>0.05$.

\subsection{Numerical Results for Electron Acceleration}

In this section we present numerical results for electron acceleration by solving the following equations of motion for a test electron.

The time is normalized by the electron cyclotron period $\omega^{-1}$ ce $\left(\tilde{t}=\omega_{c e} t, \omega_{c e}=e B_{0} / m_{e} c\right)$. We take $R_{e}=10^{-8}$ and $v_{\text {ap }} / c=1 / 4300$. The initial conditions for the electron are $x(0)=y(0)=z(0)=0.5, P_{x}=P y=P_{z}=10^{-4}$. The electromagnetic fields are taken as the same one for the proton's case. Figure 4 shows the time history of the electron Lorentz factor $\Gamma_{e}$ and $P_{x} / m_{e} c$. As seen in the lower part of Figure 4, the electron can be accelerated to the negative $x$-direction opposite the proton(see Figure 3). From the numerical calculation we find the time dependency of the average momentum $\mathrm{P}_{\mathrm{x}}$ as

$$
\left|\tilde{P}_{x}\right|=2.5 \times 10^{-4} \omega_{c e} t+10^{-4}
$$

for $y(0)=0.5$,

$$
\left|\tilde{P}_{x}\right|=1.0 \times 10^{-5} \omega_{c e} t+10^{-4}
$$

for $y(0)=0.1$ and

$$
\left|\tilde{P}_{x}\right|=4.0 \times 10^{-6} \omega_{c e} t+10^{-4},
$$

for $y(0)=0.05$. These results are almost same for the initial momentum in the range of $P(0)=10^{-6} 1010^{-4}$ as well as for the other initial positions, $x(0)$ and $z(0)$. As the electron cyclotron frequency is $(1) \mathrm{ce}=10^{8} \mathrm{~s}^{-1}$ for $\mathrm{B}_{0}=10 \mathrm{G}$, 
we conclude from Equation (38) that within $1 \mathrm{~s}$ the electron with $\mathrm{y}(0)>0.05$ can be accelerated up to $\sim 200 \mathrm{MeV}$.

\section{Summary}

In this paper, we presented a model for high-energy solar flares to explain prompt proton and electron acceleration, which occurs around moving $X$-point magnetic field. We derived the non-stationary electromagnetic fields during the strong implosion of the current sheet, which is driven by the converging flow toward the center of the magnetic arcade. We investigated a test particle motion in the electromagnetic fields derived from the MHD equations. It has been shown that both protons and electrons can be promptly(within $1 \mathrm{~s}$ ) accelerated up to $\sim 70 \mathrm{MeV}$ and $\sim 200$ $\mathrm{MeV}$, respectively. This acceleration process can be applicable for the impulsive phase of the gradual gamma ray and proton flares(gradual GR/P flare), which have been called two-ribbon flares.

\section{Acknowlegements}

The author would like to thank Mr. H. Ame of the ELCO Company for his scientific financial support. This work is supported in part by a Grant-in Aid for Scientific Research from the Ministry of Education (03640247).

\section{References}

Bai, T., and Sturrock, P. A. : 1989, Ann. Rev. Astr. Astrophys. 27, 421.

De Jager, C., and Sakai, J.-I. : 1991, Solar Phys. 133, 395.

Kahler, S.W., Moor, R.L., Kane, S.R., and Zirin, H.: 1988, Ap. J. 328, 824.

Kane, S. R. , Chupp, E. L., Forrest, D. J., Share, G. H., and Rieger, E. : 1986, Ap. J. (Letters) 300, L95

Martin, S.F., and Ramsey, H.E.: 1972, in Solar Activity Observations and Predictions, ed. P.S. McIntosh and M. Dryer (Cambridge; M.I.T. Press), p.371.

Rieger, E. : 1989, Solar Phys. 121, 323.

Sakai, J.-I. : 1990 , Ap. J. 365, 354.

Sakai, J.-I., and Koide, S. : 1991, Research Report on Plasma Astrophysics and Fusion Science, PAFS-2, June 1991 (Toyama University), to appear in Solar Physics.

Sakai, J.-I., and Ohsawa, Y.: 1987, Space Sci. Rev. 46, 143.

Sakai, J.-I., Tajima, T., and Brunel, F. : 1984, Solar Phys. 91, 103.

Tajima,T. : 1982, in Fusion Energy-1981, International Center for Theoretical Physics, Trieste, 1982, p.403.
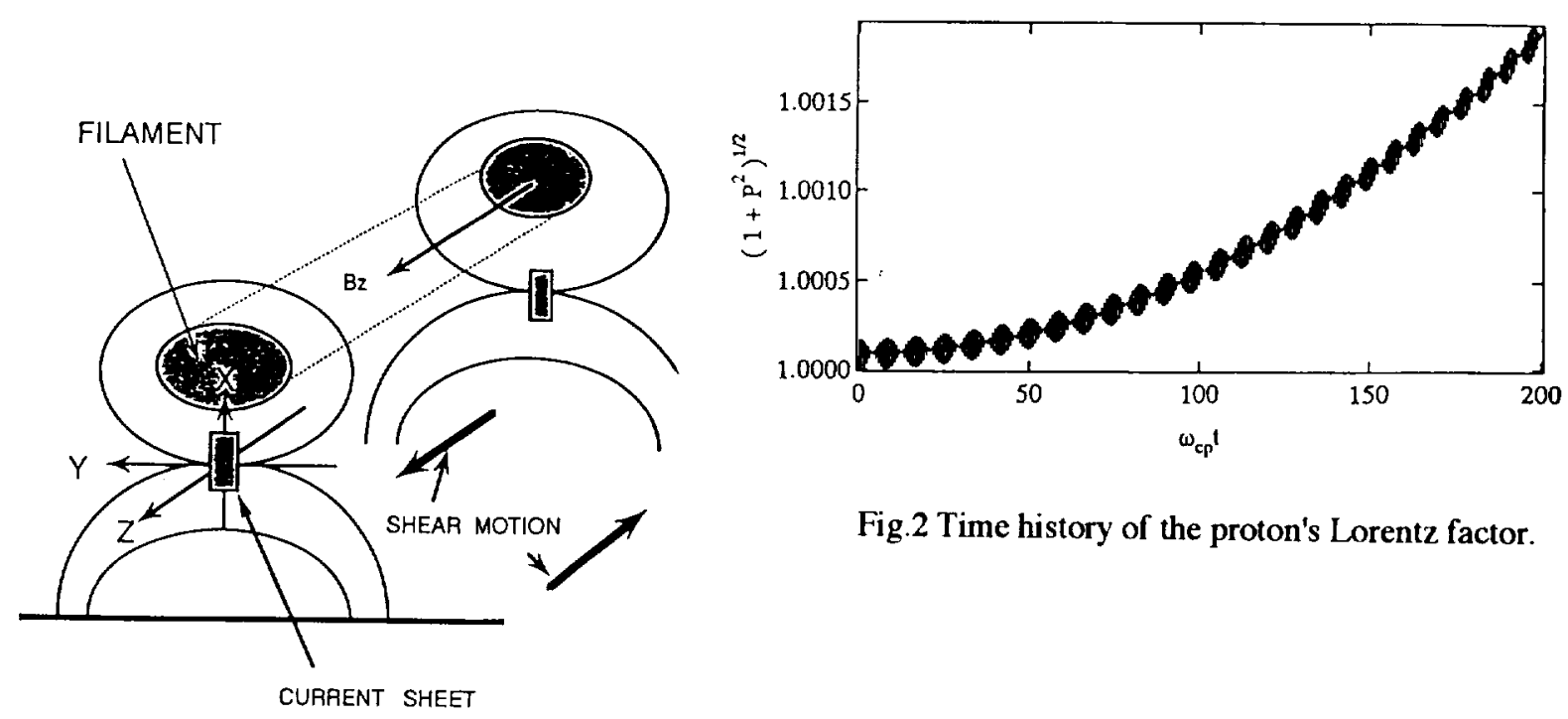

Fig. 2 Time history of the proton's Lorentz factor.

Fig.1 Schematic magnetic configuration and coordinate system. 


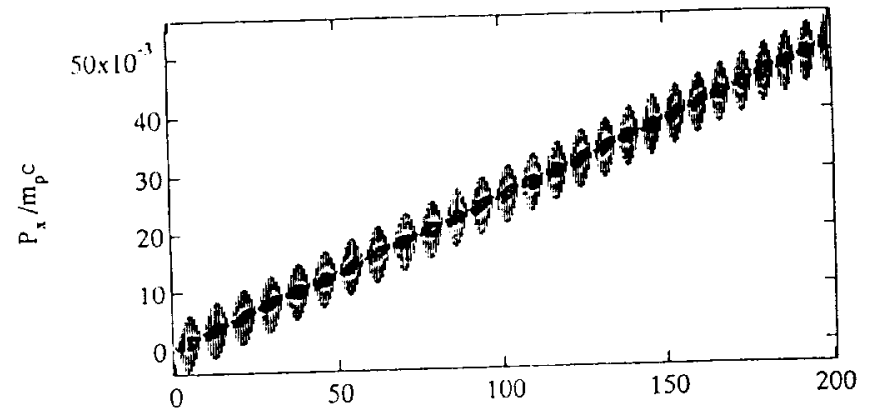

Fig.3 Time history of the proton's momentum.
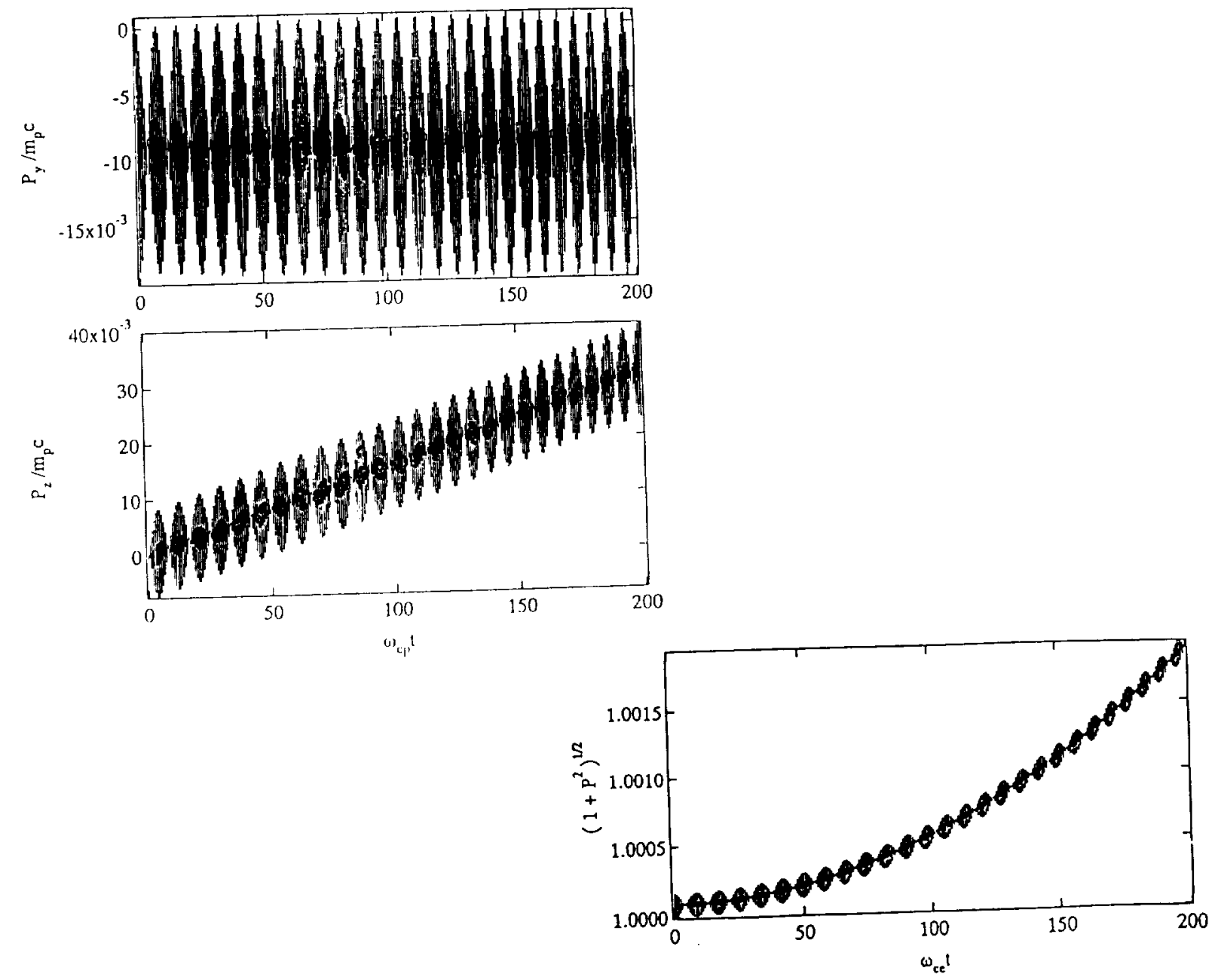

Fig.4 Time history of the electron's Lorentz. factor(upper) and momentum $P_{x}$ (lower).

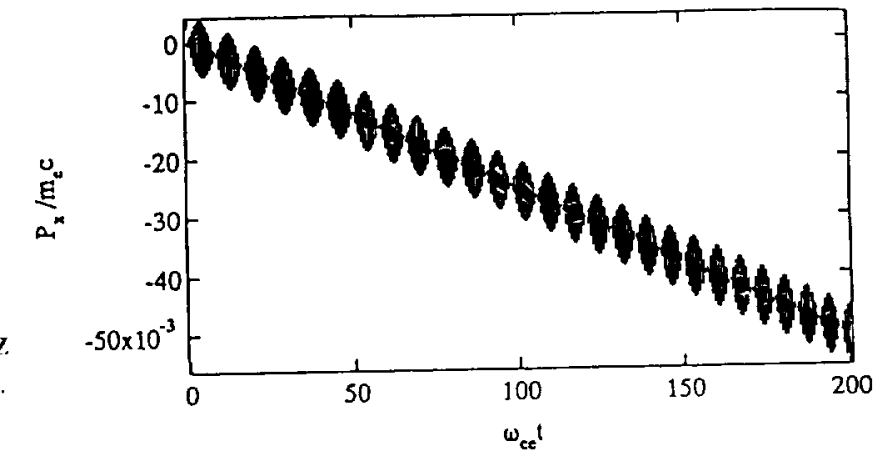

